\title{
Resting heart rate and all-cause and cardiovascular mortality in the general population: a meta-analysis
}

\author{
Dongfeng Zhang MD PhD, Xiaoli Shen MD PhD, Xin Qi MD PhD
}

\begin{abstract}
Background: Data on resting heart rate and risk of all-cause and cardiovascular mortality are inconsistent; the magnitude of associations between resting heart rate and risk of all-cause and cardiovascular mortality varies across studies. We performed a meta-analysis of prospective cohort studies to quantitatively evaluate the associations in the general population.
\end{abstract}

Methods: We searched PubMed, Embase and MEDLINE from inception to Jan. 1, 2015. We used a random-effects model to combine study-specific relative risks and $95 \%$ confidence intervals (Cls). We used restricted cubic spline functions to assess the dose-response relation.

Results: A total of 46 studies were included in the meta-analysis, involving 1246203 patients and 78349 deaths for all-cause mortality, and 848320 patients and 25800 deaths for cardiovascular mortality. The relative risk with 10 beats/min increment of resting heart rate was $1.09(95 \% \mathrm{Cl} 1.07-1.12)$ for all-cause mortality and 1.08 (95\% Cl 1.06-1.10) for cardiovascular mortality. Compared with the lowest category, patients with a resting heart rate of $60-80$ beats/min had a relative risk of 1.12 (95\% Cl 1.07-1.17) for all-cause mortality and 1.08 (95\% Cl 0.99-1.17) for cardiovascular mortality, and those with a resting heart rate of greater than 80 beats $/ \mathrm{min}$ had a relative risk of $1.45(95 \% \mathrm{Cl} 1.34-1.57)$ for all-cause mortality and $1.33(95 \% \mathrm{Cl} 1.19-1.47)$ for cardiovascular mortality. Overall, the results did not differ after adjustment for traditional risk factors for cardiovascular disease. Compared with 45 beats/min, the risk of all-cause mortality increased significantly with increasing resting heart rate in a linear relation, but a significantly increased risk of cardiovascular mortality was observed at 90 beats/min. Substantial heterogeneity and publication bias were detected.

Interpretation: Higher resting heart rate was independently associated with increased risks of all-cause and cardiovascular mortality. This indicates that resting heart rate is a predictor of all-cause and cardiovascular mortality in the general population.
$\mathrm{R}$ esting heart rate is a familiar, straightforward and inexpensive-to-measure clinical variable, and it can be modified by a number of factors, such as physical activity, psychologic stress, smoking, intake of omega-3 fatty acids and medications. ${ }^{1-4}$ Higher heart rate has been linked to increased risks of cardiovascular disease and premature mortality through a multitude of actions, including its detrimental effects on progression of coronary atherosclerosis, on occurrence of myocardial ischemia and ventricular arrhythmia, on left ventricular function and on circulating levels of inflammatory markers. ${ }^{5-7}$ However, measurement of resting heart rate has not been recommended in American and European guidelines on risk assessment of cardiovascular disease. ${ }^{8,9}$ The major reasons for this may be that the magnitude of risk with each increment of higher resting heart rate has been inconsistent among studies, and that rest- ing heart rate may not be an independent predictor because higher heart rate coexists with traditional risk factors of cardiovascular disease. ${ }^{10-13}$ In addition, data are lacking on the dose-response relation between resting heart rate and all-cause and cardiovascular mortality, ${ }^{5,9}$ although a previous review showed a continuous increase in risk with resting heart rate above 60 beats $/$ min. $^{5}$

Therefore, we conducted a meta-analysis of prospective cohort studies involving the general population, following the MOOSE checklist, ${ }^{14}$ to assess the risk of all-cause and cardiovascular mortality associated with each increment of 10 beats/min; to evaluate the possible doseresponse relation by restricted cubic spline functions and by pooling risks of all-cause and cardiovascular mortality for comparable categories of resting heart rate $(60-80$ beats/min and $>80$ beats $/ \mathrm{min}$ ); and to evaluate the effect of
Competing interests: None declared.

This article has been peer reviewed.

Accepted: Sept. 17, 2015 Online: Nov. 23, 2015

Correspondence to: Dongfeng Zhang, zhangdf1961@126.com

CMAJ 2016. DOI:10.1503/ cmaj.150535 
traditional risk factors of cardiovascular disease on the association of resting heart rate with risk of all-cause and cardiovascular mortality.

\section{Methods}

\section{Literature search and selection}

We searched PubMed, Embase and MEDLINE from inception to Jan. 1, 2015, restricting the search to studies involving humans and published in English or Chinese. Details of the search strategy are shown in Appendix 1 (available at www.cmaj.ca/lookup/suppl/doi:10.1503/ cmaj.150535/-/DC1). We reviewed the reference lists from retrieved articles to search for further relevant studies.

Studies were included if they met the following criteria: the study group was the general population (i.e., excluding studies in disease-specific populations); the exposure of interest was resting heart rate; the outcome of interest was all-cause or cardiovascular mortality; age- or multivariateadjusted relative risk estimates with 95\% confidence intervals (CIs) were provided; and the study used a prospective design. If data were duplicated in more than 1 study, we included the study with the longest follow-up duration. Two investigators (D.Z. and X.S) independently conducted the literature search and selection.

\section{Data extraction}

The same 2 investigators independently extracted the following data from each study: the first author's last name, publication year, followup duration, country or region where the study was performed, number of participants and deaths, method of resting heart rate assessment, use of heart rate-lowering medication at baseline, variables adjusted for in the analysis and relative risk estimates with corresponding $95 \%$ CIs for each category of resting heart rate.

For dose-response analysis with restricted cubic spline models, we extracted the number of deaths and participants (person-years) and relative risk $(95 \% \mathrm{CI})$ for each category of resting heart rate. For each study, the median level of resting heart rate for each category was assigned to each corresponding RR estimate. If the upper boundary of the highest category was not provided, we assumed that the boundary had the same amplitude as the adjacent category. We extracted relative risk estimates that reflected the greatest degree of control for potential confounders.

\section{Statistical analysis}

Pooled measurement was calculated as the inverse variance-weighted mean of the logarithm of relative risk for an increment of 10 beats/min in rest- ing heart rate. We used a random-effects model to combine study-specific relative risks, which considers both within-study and between-study variation. ${ }^{15}$ The $l^{2}$ test was used to assess heterogeneity. ${ }^{16}$ We conducted meta-regression and subgroup analysis to explore potential sources of heterogeneity and perform comparisons between groups, and we calculated $p$ values from meta-regression with a permutation test of 1000 to control spurious findings. ${ }^{17} \mathrm{~A}$ sensitivity analysis was performed, with one study removed at a time to assess whether the results could have been affected markedly by a single study. We evaluated publication bias using the Egger regression asymmetry test. ${ }^{18}$ When publication bias was detected, we adopted the trim-and-fill method to adjust the meta-analysis. This method first "trims" the smaller studies, causing funnel plot asymmetry, and estimates the true "centre" of the funnel with the trimmed funnel plot, and then replaces the omitted studies and their missing "counterparts" around the centre (filling). ${ }^{19}$ In addition, we pooled relative risks for comparable categories of resting heart rate (6080 beats $/ \mathrm{min}$ and $>80$ beats $/ \mathrm{min}$ ) as compared with the lowest category. We assessed study quality using the 9-star Newcastle-Ottawa Scale. ${ }^{20}$

To assess the dose-response relation, we performed a 2-stage random-effects dose-response meta-analysis ${ }^{21}$ taking into account betweenstudy heterogeneity. In the first stage, a restricted cubic spline model with 3 knots at the 25th, 50th and 75th percentiles ${ }^{22}$ of the levels of resting heart rate was estimated using generalized leastsquare regression, taking into account the correlation within each set of published relative risks. ${ }^{23}$ We then combined study-specific estimates using the restricted maximum likelihood method in a multivariate random-effects metaanalysis. ${ }^{24} \mathrm{~A} p$ value for nonlinearity was calculated by testing the null hypothesis that the coefficient of the second spline is equal to 0 . We used 45 beats/min (the lowest value in the included studies) as the reference. In addition, 70 beats/ min was then adopted as the reference to examine the potentially protective effect by lower values of heart rate (45-69 beats/min). All statistical analyses were performed with STATA version 12.0. All reported probabilities ( $p$ values) were 2 -sided, with $p<0.05$ considered significant.

\section{Results}

\section{Search results and study characteristics}

After a review of titles and abstracts of the records identified through the literature search, 185 potentially eligible records were identified. After a review of the full texts, 139 records were excluded (Appendix 1). Of the 46 prospective 
Table 1: Characteristics of studies included in the meta-analysis

\begin{tabular}{|c|c|c|c|c|c|c|}
\hline Study & $\begin{array}{l}\text { Country/ } \\
\text { region }\end{array}$ & Age, yr (sex) & No. patients (type of mortality) & $\begin{array}{l}\text { Follow- } \\
\text { up, yr }\end{array}$ & $\begin{array}{l}\text { Resting heart rate } \\
\text { assessment }\end{array}$ & $\begin{array}{l}\text { Study } \\
\text { quality* }\end{array}$ \\
\hline Gillum et al., $1991^{25}$ & US & $\begin{array}{l}61.6 \text { (men) } \\
62.5 \text { (women) }\end{array}$ & $\begin{array}{c}\text { Men: } 2774 \text { (all-cause: 776; CVD: 373) } \\
\text { Women: } 3221 \text { (all-cause: 544; CVD: 265) }\end{array}$ & 10.1 & Palpation & 8 \\
\hline Sandvik et al., $1993^{26}$ & Norway & 49.7 (men) & 1960 (all-cause: 271; CVD: 144) & 16 & ECG & 7 \\
\hline Mensink et al., $1997^{27}$ & Germany & $40-80$ (both) & $\begin{array}{c}\text { Men: } 1827 \text { (all-cause: 205; CVD: 85) } \\
\text { Women: } 2929 \text { (all-cause: 207; CVD: 68) }\end{array}$ & 12 & ECG & 7 \\
\hline Benetos et al., $1999^{28}$ & France & $\begin{array}{l}51.1 \text { (men) } \\
52.1 \text { (women) }\end{array}$ & $\begin{array}{l}\text { Men: } 12123 \text { (all-cause: 2036; CVD: 664) } \\
\text { Women: } 7263 \text { (all-cause: 610; CVD: 180) }\end{array}$ & 18.2 & ECG & 7 \\
\hline Greenland et al., $1999^{29}$ & US & $\begin{array}{l}39.8 \text { (men) } \\
40.1 \text { (women) }\end{array}$ & $\begin{array}{c}\text { Men: } 18787 \text { (all-cause: 3384) } \\
\text { Women: } 14994 \text { (all-cause: 1693) }\end{array}$ & 22 & ECG & 9 \\
\hline Palatine et al., $1999^{30}$ & US & 72.7 (men) & 763 (all-cause: 408; CVD: 200) & 12 & Palpation & 6 \\
\hline $\begin{array}{l}\text { Kristal-Boneh et al., } \\
2000^{31}\end{array}$ & Israel & 45.6 (men) & 3537 (all-cause: 135; CVD: 57) & 8 & ECG & 6 \\
\hline Reunanen et al., $2000^{32}$ & Finland $^{3}$ & 30-59 (both) & $\begin{array}{l}\text { Men: } 5598 \text { (all-cause: 1848; CVD: } 1033 \\
\text { Women: } 5119 \text { (all-cause: 840; CVD: 426) }\end{array}$ & 23 & ECG & 7 \\
\hline Nilsson et al., $2001^{33}$ & Sweden & $\begin{array}{l}49.6 \text { (men) } \\
43.7 \text { (women) }\end{array}$ & $\begin{array}{l}\text { Men: } 22444 \text { (all-cause: 1902; CVD: 727) } \\
\text { Women: } 10902 \text { (all-cause: 397; CVD: 85) }\end{array}$ & $\begin{array}{l}17 \\
12\end{array}$ & $\begin{array}{l}\text { A device while } \\
\text { measuring } \mathrm{BP}\end{array}$ & 9 \\
\hline Seccareccia et al., $2001^{34}$ & Italy & $40-69$ (men) & 2533 (all-cause: 350; CVD: 133) & 8.5 & ECG & 6 \\
\hline Cheng et al., $2002^{35}$ & US & 40.8 (men) & 27459 (all-cause: 724; CVD: 205) & 13.0 & ECG & 8 \\
\hline Kado et al., $2002^{36}$ & US & 71.7 (women) & 9702 (all-cause: 1947) & 8.9 & Palpation & 6 \\
\hline Chang et al., $2003^{37}$ & US & 78 (women) & 942 (all-cause: not reported) & 3 & ECG & 5 \\
\hline Hozawa et al., $2004^{38}$ & Japan & 60.3 (both) & 1780 (CVD: not reported) & 10 & $\begin{array}{l}\text { A device while } \\
\text { measuring } \mathrm{BP}\end{array}$ & 7 \\
\hline Okamura et al., $2004^{39}$ & Japan & $\begin{array}{l}49.6 \text { (men) } \\
50.0 \text { (women) }\end{array}$ & $\begin{array}{l}\text { Men: } 4640 \text { (all-cause: 875; CVD: 291) } \\
\text { Women: } 5906 \text { (all-cause: 731; CVD: 276) }\end{array}$ & 16.5 & ECG & 8 \\
\hline Fisher et al., $2006^{40}$ & Australia & 83.2 (both) & 179 (all-cause: 97 ) & 5 & $\begin{array}{l}\text { A device while } \\
\text { measuring BP }\end{array}$ & 5 \\
\hline Savonen et al., $2006^{41}$ & Finland & 54 (men) & 1378 (all-cause: 146; CVD: 56) & 11.4 & ECG & 8 \\
\hline Theobald et al., $2007^{42}$ & Sweden & $\begin{array}{l}37.6 \text { (men) } \\
33.3 \text { (women) }\end{array}$ & $\begin{array}{l}\text { Men: } 490 \text { (all-cause: 120) } \\
\text { Women: } 499 \text { (all-cause: 84) }\end{array}$ & 26 & Palpation & 7 \\
\hline Adabag et al., $2008^{43}$ & US & 36 (men) & 12555 (all-cause: 4642) & 25 & ECG & 8 \\
\hline Hansen et al., $2008^{44}$ & Multiple & 56.2 (both) & 6928 (all-cause: 850; CVD: 325) & 9.6 & Palpation & 5 \\
\hline Kizilbash et al., $2008^{45}$ & US & $\begin{array}{l}38.4 \text { (men) } \\
36.3 \text { (women) }\end{array}$ & $\begin{array}{c}\text { Men: } 3918 \text { (CVD: 496) } \\
\text { Women: } 8664 \text { (CVD: 527) }\end{array}$ & 32 & ECG & 8 \\
\hline Tverdal et al., $2008^{13}$ & Norway & $\begin{array}{l}41.4 \text { (men) } \\
41.4 \text { (women) }\end{array}$ & $\begin{array}{c}\text { Men: } 180353 \text { (all-cause: 5056; CVD: 1283) } \\
\text { Women: } 199490 \text { (all-cause: 3895; CVD: 460) }\end{array}$ & 12 & $\begin{array}{l}\text { A device while } \\
\text { measuring BP }\end{array}$ & 8 \\
\hline Mai et al., $2009^{46}$ & China & 47 (both) & 9856 (all-cause: 1523) & 16.2 & ECG & 7 \\
\hline Batty et al., $2010^{47}$ & UK & 40-69 (men) & 1183 (all-cause: 940 ) & 40 & Unclear & 5 \\
\hline Cooney et al., $2010^{12}$ & Finland & $\begin{array}{l}43.3 \text { (men) } \\
43.0 \text { (women) }\end{array}$ & $\begin{array}{l}\text { Men: } 10519 \text { (CVD: 266; all-cause: 806) } \\
\text { Women: } 11334 \text { (CVD: 96; all-cause: 408) }\end{array}$ & 12 & Palpation & 8 \\
\hline Nauman et al., $2010^{48}$ & Norway & $\begin{array}{l}46 \text { (men) } \\
46 \text { (women) }\end{array}$ & $\begin{array}{c}\text { Men: } 24999 \text { (CVD: 2566) } \\
\text { Women: } 25089 \text { (CVD: 1814) }\end{array}$ & 18.2 & Palpation & 7 \\
\hline Jensen et al., $2011^{49}$ & Denmark & 49.8 (both) & 16516 (all-cause: 8709; CVD: 3821) & 21.2 & ECG & 8 \\
\hline Jouven et al., $2011^{50}$ & France & 48.0 (men) & 6101 (all-cause: 1635; CVD: 435) & 25 & Palpation & 6 \\
\hline Legeai et al., $2011^{51}$ & France & 73.9 (both) & 7147 (all-cause: 615; CVD: 110) & 6 & $\begin{array}{l}\text { A device while } \\
\text { measuring BP }\end{array}$ & 5 \\
\hline Nabi et al., $2011^{52}$ & UK & 61 (both) & 5936 (all-cause: 170) & 5.6 & ECG & 5 \\
\hline Nauman et al., $2011^{53}$ & Norway & 51.9 (both) & 29325 (all-cause: 3038) & 12 & Palpation & 9 \\
\hline Inoue et al., $2012^{54}$ & Japan & 59 (both) & 2583 (all-cause: 454; CVD: 153) & 12.0 & $\begin{array}{l}\text { A device while } \\
\text { measuring } \mathrm{BP}\end{array}$ & 7 \\
\hline Leistner et al., $2012^{10}$ & Germany & 55.9 (both) & 5320 (all-cause: 137; CVD: 22) & 5 & $\begin{array}{l}\text { A device while } \\
\text { measuring BP }\end{array}$ & 6 \\
\hline $\begin{array}{l}\text { Woodward et al., } \\
2012^{55}\end{array}$ & $\begin{array}{l}\text { Asia-Pacific } \\
\text { region }\end{array}$ & 51 (both) & 112680 (all-cause: 6086; CVD: 2055) & 7.4 & Unclear & 6 \\
\hline Jensen et al., $2013^{56}$ & Denmark & 62.6 (men) & 2798 (all-cause: 1082) & 16 & ECG & 7 \\
\hline Johansen et al., $2013^{57}$ & Denmark & 64.4 (both) & 653 (all-cause: 80 ) & 6.3 & ECG & 7 \\
\hline Pittaras et al., $2013^{58}$ & US & 58 (men) & 18642 (all-cause: 5100 ) & 10 & ECG & 7 \\
\hline Plichart et al., $2013^{59}$ & France & $\begin{array}{l}44.6 \text { (men) } \\
44.9 \text { (women) }\end{array}$ & $\begin{array}{l}\text { Men: } 141533 \text { (all-cause: not reported) } \\
\text { Women: } 84755 \text { (all-cause: not reported) }\end{array}$ & 5 & ECG & 6 \\
\hline Saxena et al., $2013^{60}$ & US & 44.0 (both) & 53322 (all-cause: 3125; CVD: 1081) & 15.0 & ECG & 8 \\
\hline Aladin et al., $2014^{61}$ & US & 53.0 (both) & 56634 (all-cause: 6255) & 11.1 & Unclear & 7 \\
\hline Floyd et al., $2014^{62}$ & US & 76 (both) & 1991 (all-cause: 1326) & 12 & ECG & 7 \\
\hline Hisamatsu et al., $2014^{63}$ & Japan & 48.7 (both) & 8807 (CVD: 617) & 24 & ECG & 7 \\
\hline Ho et al., $2014^{64}$ & US & 55 (both) & 4058 (all-cause: 1186; CVD: 252) & 19 & ECG & 7 \\
\hline Ó Hartaigh et al., $2014^{65}$ & US & 43 (both) & 2865 (all-cause: 246 ) & 23 & Palpation & 6 \\
\hline Ryu et al., $2014^{66}$ & Korea & 66.2 (men) & 2600 (all-cause: 1990; CVD: 337) & 20.8 & Palpation & 7 \\
\hline Wang et al., $2014^{67}$ & China & 51.1 (both) & 92562 (all-cause: 1589) & 4 & ECG & 7 \\
\hline
\end{tabular}


Table 2: Pooled results on resting heart rate and risk of all-cause mortality

\begin{tabular}{|c|c|c|c|c|c|c|c|c|c|c|c|c|}
\hline \multirow[b]{2}{*}{ Variable } & \multicolumn{4}{|c|}{$60-80$ beats/min } & \multicolumn{4}{|c|}{$>80$ beats $/ \mathrm{min}$} & \multicolumn{4}{|c|}{10 beats/min increment } \\
\hline & No. & RR $(95 \% \mathrm{Cl})$ & $\begin{array}{l}R^{2} \text { value, } \\
\%\end{array}$ & $p$ value & No. & RR $(95 \% \mathrm{Cl})$ & $\begin{array}{l}R^{2} \text { value, } \\
\%\end{array}$ & $p$ value & No. & RR $(95 \% \mathrm{Cl})$ & $\begin{array}{c}R^{2} \text { value, } \\
\%\end{array}$ & $p$ value \\
\hline Age-adjusted & 16 & $1.21(1.14-1.29)$ & 65.4 & & 17 & $1.66(1.48-1.87)$ & 83.9 & & 20 & $1.14(1.09-1.18)$ & 97.5 & \\
\hline Multivariate-adjusted & 25 & $1.12(1.07-1.17)$ & 66.7 & & 32 & $1.45(1.34-1.57)$ & 81.3 & & 35 & $1.09(1.07-1.12)$ & 92.3 & \\
\hline \multicolumn{9}{|c|}{ Excluding deaths during the first $6 \mathrm{mo}$ to $5 \mathrm{yr}$} & 12 & $1.09(1.06-1.13)$ & 85.9 & \\
\hline \multicolumn{9}{|c|}{ Among those not taking heart rate-lowering medication } & 12 & $1.11(1.06-1.16)$ & 95.3 & \\
\hline \multicolumn{9}{|c|}{ Among studies adjusting for heart rate-lowering medication } & 8 & $1.13(1.07-1.19)$ & 94.4 & \\
\hline \multicolumn{4}{|c|}{$\begin{array}{l}\text { Among studies adjusting for heart rate-lowering medication } \\
\text { Follow-up duration }\end{array}$} & 0.5 & & & & 0.01 & & & & 0.08 \\
\hline$>10 \mathrm{yr}$ & 15 & $1.11(1.04-1.18)$ & 76.0 & & 18 & $1.34(1.22-1.48)$ & 82.5 & & 27 & $1.08(1.06-1.10)$ & 90.5 & \\
\hline$\leq 10 \mathrm{yr}$ & 10 & $1.14(1.08-1.20)$ & 0.00 & & 14 & $1.64(1.51-1.78)$ & 40.7 & & 8 & $1.14(1.07-1.22)$ & 93.4 & \\
\hline Sample size & & & & 0.1 & & & & 0.8 & & & & 0.8 \\
\hline$>10000$ & 10 & $1.07(1.02-1.13)$ & 67.2 & & 12 & $1.43(1.30-1.57)$ & 83.4 & & 15 & $1.10(1.06-1.14)$ & 96.0 & \\
\hline$\leq 10000$ & 15 & $1.17(1.08-1.27)$ & 55.0 & & 20 & $1.48(1.29-1.70)$ & 80.3 & & 20 & $1.08(1.06-1.10)$ & 78.3 & \\
\hline Mean age, yr & & & & 0.3 & & & & 0.8 & & & & 0.03 \\
\hline$>50$ & 16 & $1.15(1.08-1.22)$ & 59.5 & & 19 & $1.46(1.33-1.60)$ & 73.8 & & 18 & $1.13(1.09-1.17)$ & 90.6 & \\
\hline$\leq 50$ & 9 & $1.08(1.00-1.16)$ & 72.5 & & 13 & $1.44(1.24-1.66)$ & 87.5 & & 17 & $1.07(1.04-1.09)$ & 92.2 & \\
\hline Region & & & & 0.2 & & & & 0.5 & & & & 0.04 \\
\hline Europe & 14 & $1.18(1.10-1.27)$ & 55.2 & & 17 & $1.52(1.33-1.74)$ & 83.3 & & 18 & $1.09(1.06-1.11)$ & 87.7 & \\
\hline United States & 7 & $1.04(0.99-1.10)$ & 59.1 & & 9 & $1.39(1.22-1.57)$ & 80.2 & & 9 & $1.05(1.03-1.08)$ & 85.8 & \\
\hline Asia & 3 & $1.05(0.84-1.30)$ & 70.6 & & 4 & $1.35(1.11-1.64)$ & 70.3 & & 6 & $1.13(1.07-1.19)$ & 62.5 & \\
\hline Mixed & 1 & $1.17(1.04-1.31)$ & - & & 1 & $1.54(1.43-1.66)$ & - & & 2 & $1.25(1.01-1.56)$ & 95.2 & \\
\hline Australia & - & - & - & & 1 & $1.19(0.45-3.16)$ & - & & & & & \\
\hline Excluding CVD at baseline & & & & 0.2 & & & & 0.07 & & & & 0.6 \\
\hline Yes & 16 & $1.10(1.03-1.16)$ & 72.9 & & 18 & $1.36(1.24-1.50)$ & 82.8 & & 23 & $1.09(1.06-1.12)$ & 92.2 & \\
\hline No & 9 & $1.15(1.09-1.21)$ & 0.00 & & 14 & $1.60(1.42-1.80)$ & 70.3 & & 12 & $1.11(1.07-1.15)$ & 92.0 & \\
\hline Sex & & & & 0.9 & & & & $>0.9$ & & & & 0.1 \\
\hline Male & 12 & $1.13(1.06-1.21)$ & 68.6 & & 14 & $1.47(1.27-1.71)$ & 89.0 & & 14 & $1.08(1.05-1.11)$ & 91.6 & \\
\hline Female & 4 & $1.06(0.96-1.19)$ & 26.7 & & 7 & $1.39(1.18-1.65)$ & 59.7 & & 9 & $1.07(1.04-1.10)$ & 76.5 & \\
\hline Both sexes & 9 & $1.13(1.03-1.24)$ & 73.5 & & 11 & $1.44(1.31-1.59)$ & 68.0 & & 12 & $1.14(1.08-1.20)$ & 95.6 & \\
\hline Resting heart rate assessment & & & & $>0.9$ & & & & 0.4 & & & & 0.01 \\
\hline Electrocardiogram & 12 & $1.13(1.04-1.22)$ & 70.1 & & 17 & $1.52(1.35-1.70)$ & 79.2 & & 23 & $1.06(1.04-1.08)$ & 89.0 & \\
\hline Others & 13 & $1.12(1.05-1.19)$ & 64.7 & & 15 & $1.40(1.25-1.56)$ & 84.1 & & 12 & $1.15(1.10-1.20)$ & 89.6 & \\
\hline Minimum of resting period & & & & $>0.9$ & & & & 0.2 & & & & 0.9 \\
\hline$\geq 5 \mathrm{~min}$ & 8 & $1.13(0.99-1.29)$ & 80.8 & & 13 & $1.60(1.38-1.85)$ & 82.0 & & 19 & $1.09(1.06-1.12)$ & 89.5 & \\
\hline$<5$ min or unclear & 17 & $1.11(1.06-1.16)$ & 54.7 & & 19 & $1.39(1.27-1.51)$ & 75.5 & & 16 & $1.10(1.06-1.14)$ & 94.5 & \\
\hline Posture & & & & 0.2 & & & & 0.2 & & & & 0.8 \\
\hline Supine & 9 & $1.19(1.06-1.35)$ & 78.7 & & 15 & $1.56(1.32-1.84)$ & 86.8 & & 13 & $1.09(1.05-1.13)$ & 92.8 & \\
\hline Sitting/ recumbent/unclear & 16 & $1.09(1.04-1.14)$ & 52.0 & & 17 & $1.36(1.26-1.47)$ & 70.8 & & 22 & $1.10(1.07-1.12)$ & 92.2 & \\
\hline Study quality* & & & & 0.05 & & & & 0.01 & & & & 0.4 \\
\hline $7-9$ & 16 & $1.08(1.03-1.14)$ & 66.8 & & 18 & $1.33(1.24-1.44)$ & 73.2 & & 28 & $1.09(1.06-1.11)$ & 91.3 & \\
\hline $5-6$ & 9 & $1.22(1.14-1.31)$ & 8.60 & & 14 & $1.67(1.45-1.92)$ & 76.0 & & 7 & $1.12(1.05-1.20)$ & 93.8 & \\
\hline Adjusted for ... & & & & & & & & & & & & \\
\hline Blood pressure & & & & 0.1 & & & & 0.09 & & & & 0.7 \\
\hline Yes & 23 & $1.11(1.06-1.16)$ & 61.8 & & 29 & $1.42(1.32-1.53)$ & 78.8 & & 34 & $1.10(1.07-1.12)$ & 92.5 & \\
\hline No & 2 & $1.22(0.85-1.75)$ & 61.0 & & 3 & $1.97(1.64-2.37)$ & 13.6 & & 1 & $1.07(1.02-1.12)$ & - & \\
\hline Smoking & & & & - & & & & 0.7 & & & & 0.8 \\
\hline Yes & 25 & $1.12(1.07-1.17)$ & 66.7 & & 31 & $1.45(1.34-1.57)$ & 81.9 & & 32 & $1.09(1.07-1.12)$ & 92.2 & \\
\hline No & 0 & - & - & & 1 & $1.19(0.45-3.18)$ & - & & 1 & $1.11(1.08-1.14)$ & - & \\
\hline Body mass index & & & & 0.6 & & & & 0.8 & & & & 0.2 \\
\hline Yes & 19 & $1.13(1.07-1.20)$ & 65.5 & & 25 & $1.44(1.32-1.57)$ & 78.6 & & 29 & $1.09(1.06-1.11)$ & 92.2 & \\
\hline No & 6 & $1.10(1.09-1.20)$ & 74.6 & & 7 & $1.49(1.22-1.82)$ & 88.6 & & 6 & $1.12(1.08-1.15)$ & 57.2 & \\
\hline Physical activity & & & & 0.5 & & & & 0.3 & & & & 0.3 \\
\hline Yes & 13 & $1.15(1.06-1.24)$ & 70.9 & & 17 & $1.52(1.36-1.70)$ & 76.8 & & 14 & $1.08(1.05-1.10)$ & 93.4 & \\
\hline No & 12 & $1.10(1.03-1.17)$ & 64.2 & & 15 & $1.38(1.23-1.55)$ & 85.3 & & 21 & $1.11(1.07-1.16)$ & 91.9 & \\
\hline Serum cholesterol/triglycerides & & & & 0.4 & & & & 0.5 & & & & 0.9 \\
\hline Yes & 22 & $1.10(1.06-1.15)$ & 59.1 & & 26 & $1.43(1.33-1.54)$ & 77.7 & & 33 & $1.09(1.07-1.11)$ & 92.6 & \\
\hline No & 3 & $1.21(0.91-1.61)$ & 87.1 & & 6 & $1.50(1.04-2.17)$ & 88.5 & & 2 & $1.10(1.06-1.14)$ & 18.5 & \\
\hline Diabetes/blood glucose & & & & 0.03 & & & & 0.1 & & & & 0.6 \\
\hline Yes & 15 & $1.06(1.02-1.11)$ & 45.4 & & 18 & $1.37(1.25-1.50)$ & 80.7 & & 27 & $1.10(1.07-1.13)$ & 93.5 & \\
\hline No & 10 & $1.21(1.09-1.34)$ & 65.5 & & 14 & $1.58(1.37-1.82)$ & 78.7 & & 8 & $1.08(1.05-1.10)$ & 75.4 & \\
\hline Alcohol & & & & 0.5 & & & & 0.9 & & & & 0.6 \\
\hline Yes & 7 & $1.17(1.04-1.31)$ & 81.0 & & 9 & $1.44(1.30-1.59)$ & 56.5 & & 13 & $1.08(1.04-1.13)$ & 90.9 & \\
\hline No & 18 & $1.10(1.05-1.16)$ & 58.1 & & 23 & $1.45(1.31-1.61)$ & 85.1 & & 22 & $1.10(1.07-1.13)$ & 93.2 & \\
\hline Education/socioeconomic statu & & & & 0.3 & & & & 0.4 & & & & 0.3 \\
\hline Yes & 10 & $1.16(1.06-1.27)$ & 57.8 & & 10 & $1.37(1.19-1.58)$ & 76.3 & & 10 & $1.07(1.03-1.11)$ & 87.4 & \\
\hline No & 15 & $1.10(1.04-1.15)$ & 66.9 & & 22 & $1.49(1.36-1.64)$ & 82.3 & & 25 & $1.10(1.08-1.13)$ & 93.5 & \\
\hline No. covariates adjusted & & & & 0.8 & & & & 0.7 & & & & 0.1 \\
\hline$\geq 6$ & 10 & $1.13(1.04-1.24)$ & 72.3 & & 13 & $1.43(1.26-1.61)$ & 80.7 & & 17 & $1.07(1.04-1.10)$ & 88.9 & \\
\hline$<6$ & 15 & $1.11(1.05-1.18)$ & 64.5 & & 19 & $1.48(1.33-1.64)$ & 82.6 & & 18 & $1.12(1.08-1.15)$ & 94.0 & \\
\hline
\end{tabular}


cohort studies included in the meta-analysis, 40 studies provided the results for all-cause mortality, involving 1246203 patients and 78349 deaths, and 29 studies provided the results for cardiovascular mortality, involving 848320 patients and 25800 deaths. The followup duration ranged from 3 to 40 years. The study quality ranged from 5 to 9 stars (Appendix 2, www.cmaj.ca/lookup/suppl/doi:10.1503/ cmaj.150535/-/DC1). Details about the included studies are shown in Table 1.

\section{Quantitative synthesis}

\section{All-cause mortality}

Pooled results on resting heart rate and risk of all-cause mortality are shown in Table 2 . The multivariate-adjusted relative risk of all-cause mortality with every 10 beats/min increment was 1.09 (95\% CI 1.07-1.12) (Figure 1). Publication bias was detected $(p<0.01)$, and the corrected effect with the trim-and-fill method was 1.04 (95\% CI 1.02-1.06). No individual study had an excessive influence to the pooled effect in sensitivity analysis. The relative risk of all-cause mortality was 1.12 (95\% CI 1.07-1.17) for those with a resting heart rate of $60-80$ beats/min and 1.45 (95\% CI 1.34-1.57) for those with a resting heart rate greater than 80 beats/min.

\section{Cardiovascular mortality}

Pooled results on resting heart rate and risk of cardiovascular mortality are shown in Table 3 . The relative risk of cardiovascular mortality with

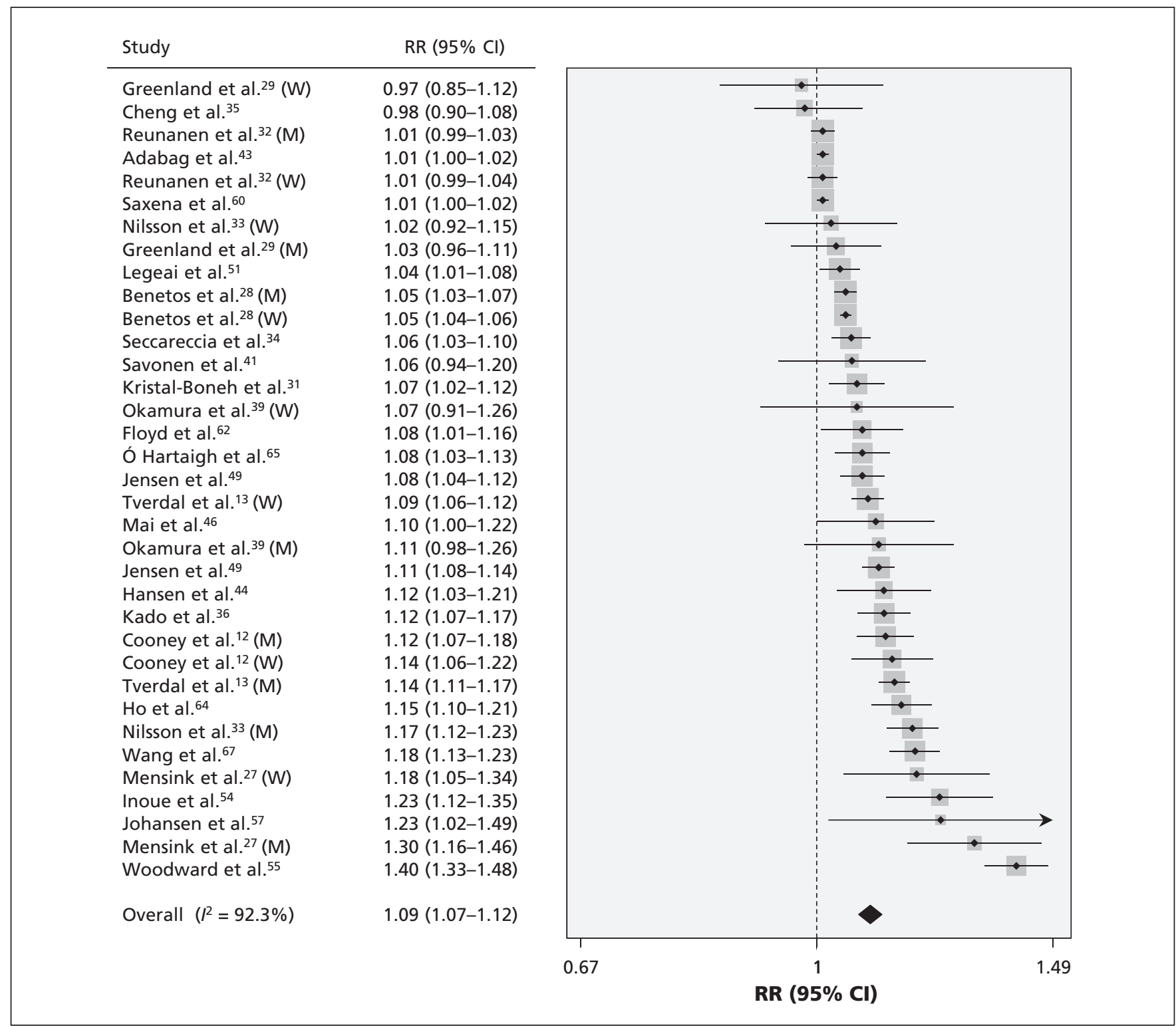

Figure 1: Risk of all-cause mortality for each 10 beats $/ \mathrm{min}$ increase in resting heart rate. Note: $\mathrm{Cl}=$ confidence interval, $\mathrm{M}=$ men, $\mathrm{RR}=$ relative risk, $\mathbf{W}=$ women. 
Table 3: Pooled results on resting heart rate and risk of cardiovascular mortality

$>80$ beats $/ \mathrm{min}$

10 beats/min increment

value, $P^{2}$ value,

$\operatorname{RR}(95 \% \mathrm{Cl}) \quad R^{2}$ value,

$\begin{array}{llllll}13 & 1.70(1.48-1.94) & 40.2 & 26 & 1.42(1.29-1.57) & 56.9\end{array}$

$\begin{array}{llllll}22 & 1.33(1.19-1.47) & 61.0 & 32 & 1.08(1.06-1.10) & 81.0\end{array}$

$1.09(1.05-1.12) \quad 67.1$

$1.13(1.07-1.19) \quad 79.0$

$1.15(1.04-1.26) \quad 88.0$

$60-80$ beats/min

Variable

Age-adjusted

Multivariate-adjusted

Excluding deaths during the first 1-5 yr

Among those not taking heart rate-lowering medication

Among studies adjusting for heart rate-lowering medication

Follow-up duration, yr

$\leq 10$
Sample size

$>10000$

$\leq 10000$

Mean age, yr

$>50$

$\leq 50$

Region

Europe

United States

Asia

Mixed

Australia

Excluding CVD at baseline

Yes

No

Sex

Male

Female

Both sexes

$\begin{array}{llll}\text { Resting heart rate assessment } & 10 & 1.10(1.00-1.22) & 27.5\end{array}$

Others

Resting period

$\geq 5$ min

$<5$ min or unclear

Posture

Supine

Sitting/ recumbent/unclear

Study quality*

7-9

5-6

Adjusted for ...

Blood pressure

Yes

No

Smoking

Yes

No

Body mass index

Yes

No

Physical activity

Yes

$10 \quad 1.13(1.01-1.28) \quad 58.7$

$\begin{array}{llll}\text { Serum cholesterol/triglycerides } & 17 & 1.06(0.98-1.15) & 41.9\end{array}$

$\begin{array}{llll}\text { Yes } & 2 & 1.18(0.74-1.88) & 91.5\end{array}$

No

Diabetes/blood glucose

Yes

No

$\begin{array}{llll}\text { Alcohol } & 3 & 1.02(0.93-1.12) & 0.00\end{array}$

$\begin{array}{llll}\text { Yes } & 16 & 1.08(0.98-1.19) & 61.0\end{array}$

$\begin{array}{llll}\text { No } & & & \\ \text { Education/socioeconomic status } & 6 & 1.04(0.93-1.17) & 6.70\end{array}$

Yes $13 \quad 1.09(0.98-1.21) \quad 65.8$

No. covariates adjusted

$\begin{array}{lccc}\geq 6 & 5 & 1.05(0.97-1.14) & 0.00 \\ <6 & 14 & 1.07(0.95-1.20) & 65.5\end{array}$

$19 \quad 1.08(0.99-1.17) \quad 55.8$
$>10$
$\leq 10$

$\begin{array}{llll}\text { Electrocardiogram } & 9 & 1.04(0.91-1.19) & 71.6\end{array}$

0.6

$\begin{array}{lll}15 & 1.26(1.11-1.44) & 68.3\end{array}$

$\begin{array}{llll}0.2 & 7 & 1.28(1.04-1.57) & 80.5\end{array}$

$15 \quad 1.35(1.20-1.53) \quad 39.6$

0.5

$12 \quad 1.25(1.06-1.46) \quad 73.3$

0.5

$12 \quad 1.28(1.04-1.58) \quad 75.9$

$6 \quad 1.42(1.27-1.58) \quad 0.00$

$3 \quad 1.25(1.02-1.52) \quad 0.00$

$7 \quad 1.15(1.04-1.26)$

75.4

0.08

0.6

$6 \quad 1.16(1.05-1.28) \quad 87.1$

$12 \quad 1.10(1.06-1.14) \quad 90.5$

$20 \quad 1.06(1.03-1.09) \quad 59.3$

0.2

$17 \quad 1.10(1.05-1.14) \quad 80.3$

0.6

$15 \quad 1.07(1.04-1.11) \quad 82.7$

$\begin{array}{lll}18 & 1.07(1.04-1.09) & 77.3\end{array}$

$1.05(1.00-1.11) \quad 70.8$

$1.11(1.02-1.21) \quad 40.9$

$2 \quad 1.27(1.00-1.60) \quad 89.4$

0.2

$1.45(1.28-1.64)$

$14 \quad 1.26(1.09-1.46) \quad 66.3$

0.3

$21 \quad 1.06(1.04-1.08) \quad 78.3$

$11 \quad 1.14(1.06-1.21) \quad 81.4$

0.6

$\begin{array}{llll}0.2 & & & \\ & 11 & 1.44(1.26-1.64) & 50.1\end{array}$

$14 \quad 1.08(1.04-1.12) \quad 73.1$

$\begin{array}{lll}9 & 1.03(1.00-1.07) & 26.2\end{array}$

$\begin{array}{lll}5 & 1.46(1.32-1.62) & 0.00\end{array}$

$\begin{array}{llll}0.5 & 10 & 1.32(1.15-1.52) & 42.2\end{array}$

$>0.9$

$12 \quad 1.33(1.13-1.56) \quad 71.2$

0.6

$11 \quad 1.43(1.29-1.58) \quad 12.0$

$19 \quad 1.05(1.03-1.08) \quad 75.5$

0.2

$13 \quad 1.14(1.08-1.21) \quad 86.2$

$\begin{array}{lll}18 & 1.08(1.04-1.11) & 70.4\end{array}$

$14 \quad 1.09(1.05-1.13) \quad 87.6$

0.4

$11 \quad 1.09(1.04-1.13) \quad 56.9$

$21 \quad 1.07(1.05-1.10) \quad 81.7$

0.05

$27 \quad 1.06(1.04-1.09) \quad 76.0$

$5 \quad 1.14(1.03-1.26) \quad 88.6$

$7 \quad 1.58(1.37-1.83) \quad 15.2$

0.1

0.4

$31 \quad 1.08(1.06-1.10) \quad 81.6$

$18 \quad 1.29(1.15-1.45) \quad 59.1$

$4 \quad 1.47(1.10-1.98) \quad 70.9$

$22 \quad 1.33(1.19-1.47) \quad 61.0$

$>0.9$

$15 \quad 1.33(1.20-1.48) \quad 39.3$

$1 \quad 1.12(1.09-1.16) \quad 0.00$

$\begin{array}{lll}7 & 1.32(1.03-1.70) & 80.2\end{array}$

$\begin{array}{lll}11 & 1.18(0.99-1.41) & 68.2\end{array}$

0.04

$27 \quad 1.08(1.05-1.10) \quad 82.1$

$5 \quad 1.08(1.03-1.14) \quad 57.0$

$\begin{array}{lll}12 & 1.04(1.02-1.06) & 80.1\end{array}$

$20 \quad 1.13(1.08-1.18) \quad 70.1$

0.6

$\begin{array}{llll}0.4 & & & \\ & 18 & 1.30(1.15-1.47) & 58.9\end{array}$

$4 \quad 1.42(1.11-1.81) \quad 74.7$

0.3

$13 \quad 1.26(1.09-1.47) \quad 67.9$

$\begin{array}{lll}9 & 1.43(1.23-1.66) & 44.9\end{array}$

0.8

$6 \quad 1.40(1.21-1.61) \quad 22.0$

$16 \quad 1.30(1.13-1.49) \quad 67.8$

0.6

$\begin{array}{lll}8 & 1.24(1.10-1.39) & 38.3\end{array}$

0.9

$14 \quad 1.41(1.20-1.66) \quad 66.4$

79.1

$21 \quad 1.09(1.05-1.13) \quad 82.2$

$10 \quad 1.04(1.02-1.07) \quad 71.9$

0.6

Note: $\mathrm{Cl}=$ confidence interval, CVD = cardiovascular disease, $\mathrm{RR}=$ relative risk

*Study quality was judged based on the Newcastle-Ottawa Scale (range 1-9 stars). 
every 10 beats/min increment was 1.08 (95\% CI 1.06-1.10) (Figure 2). Publication bias was detected $(p<0.01)$, and the corrected effect with the trim-and-fill method was 1.03 (95\% CI 1.011.06). No individual study had an excessive influence to the pooled effect in sensitivity analysis. The relative risk of cardiovascular mortality was 1.08 (95\% CI 0.99-1.17) for those with a resting heart rate of $60-80$ beats/min and 1.33 (95\% CI 1.19-1.47) for those with a rate of greater than 80 beats/min.

\section{Subgroup analysis and meta-regression}

As shown in Tables 2 and 3, between-study heterogeneity was found. Overall, no single factor successfully explained the between-study heterogeneity; however, when all of the factors were included in meta-regression simultaneously, they explained all $(100 \%)$, most $(89.2 \%, 61.9 \%)$ or part $(44.9 \%, 37.6 \%)$ of the heterogeneity (Appendix 3, www.cmaj.ca/lookup/suppl/doi:10.1503/ cmaj.150535/-/DC1).
To examine potential influencers (e.g., medication and disease states) of resting heart rate, we conducted several subgroup analyses. Among patients not taking heart rate-lowering medication, the relative risk of all-cause and cardiovascular mortality was 1.11 (95\% CI 1.06-1.16) and 1.13 (95\% CI 1.07-1.19), respectively, with each 10 beats/min increment. To take into account the possibility of an effect by an existing or preclinical disease at baseline, we conducted a separate analysis in which deaths within the first 6 months to 5 years of follow-up were excluded, and the risk of allcause mortality and cardiovascular mortality with each 10 beats/min increment was 1.09 (95\% CI 1.06-1.13) and 1.09 (95\% CI 1.051.12), respectively. For studies excluding patients with cardiovascular disease at baseline, the relative risk of all-cause mortality and cardiovascular mortality with each 10 beats/min increment was 1.09 (95\% CI 1.06-1.12) and 1.06 (95\% CI 1.04-1.08), respectively.

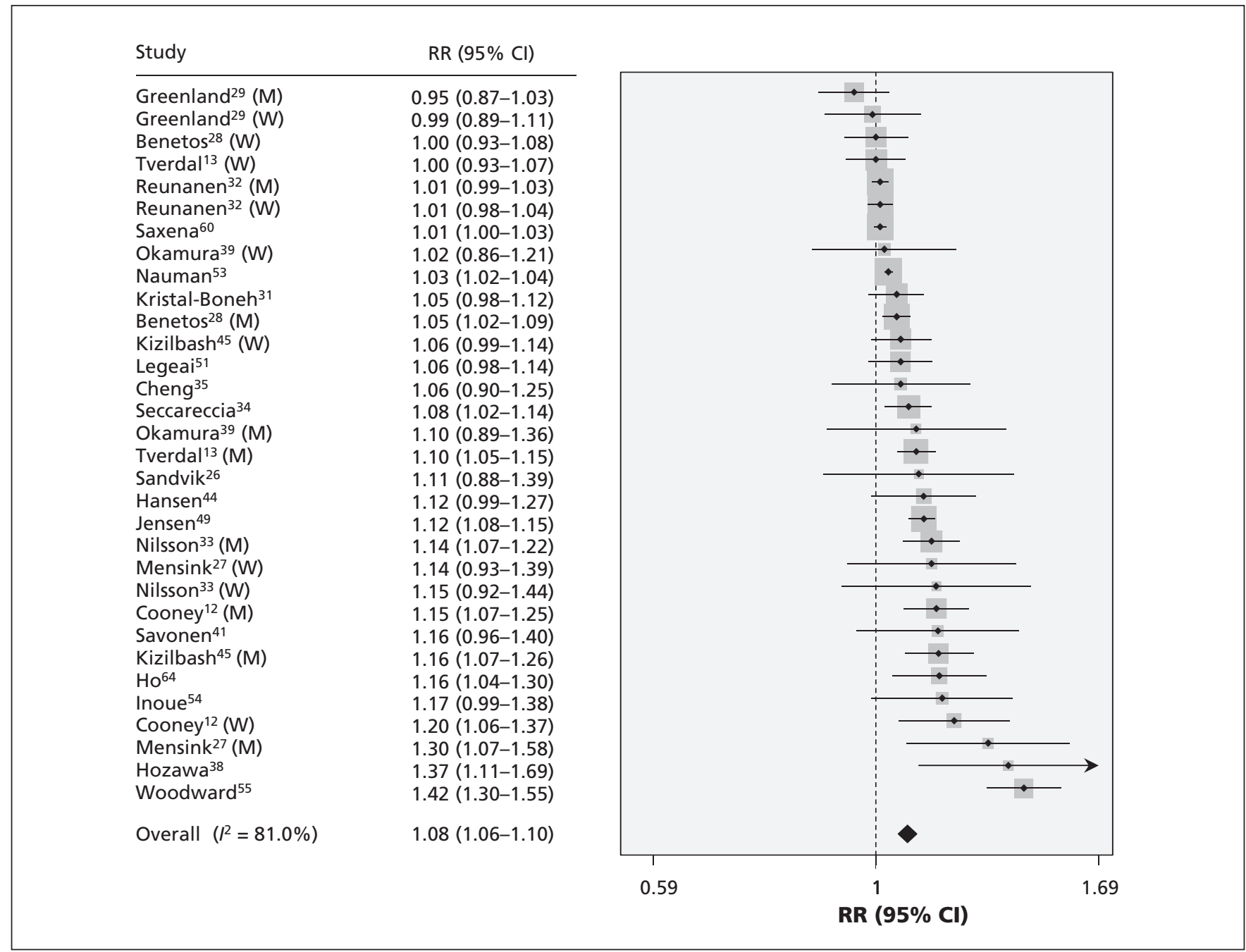

Figure 2: Risk of cardiovascular mortality for each 10 beats/min increase in resting heart rate. $\mathrm{Note}: \mathrm{Cl}=$ confidence interval, $\mathrm{M}=$ men, $\mathrm{RR}=$ relative risk, $\mathbf{W}=$ women. 


\section{Dose-response analysis with restricted cubic spline functions}

For all-cause mortality, data from 13 studies including 26636 deaths were used. The departure from a linear relation was not significant $\left(p_{\text {for nonlinearity }}=0.1\right)$. Compared with 45 beats $/ \mathrm{min}$, the risk of all-cause mortality increased significantly with increasing levels of resting heart rate (Figure 3A).

For cardiovascular mortality, data from 10 studies including 6502 deaths were used.

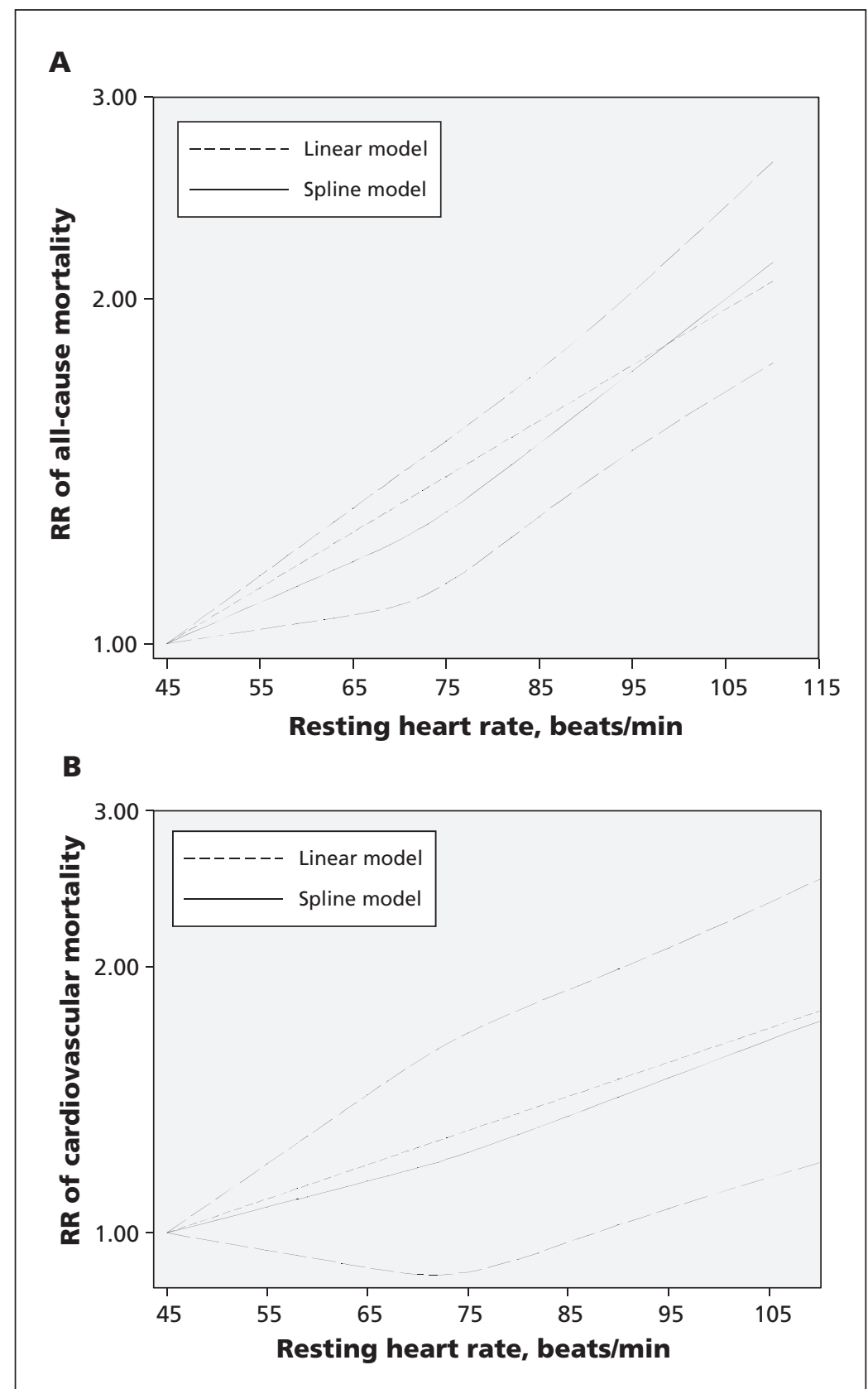

Figure 3: The dose-response analysis of resting heart rate with risk of $(A)$ allcause mortality and (B) cardiovascular mortality. The solid and long dash lines represent the estimated relative risks (RRs) and 95\% confidence intervals. The short dash lines represent the linear relation.
The departure from a linear relation was not significant $\left(p_{\text {for nonlinearity }}=0.6\right)$. Compared with 45 beats/min, the risk of cardiovascular mortality increased significantly at 90 beats/min (Figure 3B).

With use of 70 beats/min as the reference, the linear dose-response analysis indicated a protective effect of lower resting heart rate (45-69 beats/ min) on risk of all-cause and cardiovascular mortality, respectively (Appendix 4, www.cmaj.ca/ lookup/suppl/doi:10.1503/cmaj.150535/-/DC1).

\section{Interpretation}

Results from this meta-analysis suggest the risk of all-cause and cardiovascular mortality increased by $9 \%$ and $8 \%$ for every 10 beats/min increment of resting heart rate. Compared with 45 beats/min, the risk of all-cause mortality increased significantly with increasing resting heart rate in a linear relation, but a significantly increased risk of cardiovascular mortality was observed at 90 beats $/ \mathrm{min}$. The threshold (90 beats/min) associated with cardiovascular mortality is consistent with the traditionally defined tachycardia threshold of 90 or 100 beats/ min for prevention of cardiovascular disease. ${ }^{5}$

One mechanism is that higher resting heart rate is a marker of an imbalance between the vagal and the sympathetic tone, and dysfunctional autonomic nervous activity likely plays a central role in the pathogenesis of numerous adverse health conditions. ${ }^{5,68}$ A relatively high heart rate has direct detrimental effects on progression of coronary atherosclerosis, on occurrence of myocardial ischemia and ventricular arrhythmias, and on left ventricular function. In this meta-analysis, the magnitude of association between resting heart rate and all-cause mortality was stronger than that with cardiovascular mortality, and this discrepancy can be expected because resting heart rate has also been found to be associated with noncardiovascular mortality. ${ }^{28,34,44,51,54,69}$

One main concern lies in whether a high resting heart rate is an independent predictor, because higher heart rates coexist with traditional risk factors of cardiovascular disease and poor health status. ${ }^{10-13,70}$ Overall, the association of resting heart rate with risk of all-cause and cardiovascular mortality is independent of traditional risk factors of cardiovascular disease, suggesting that resting heart rate is a predictor of mortality in the general population. In addition, previous studies found that resting heart rate is also related to prognosis in settings of substantial cardiovascular comorbidities, such as renal disease, erectile dysfunction and pulmonary hypertension, as well as in noncardiovascular conditions such as chronic obstructive pulmonary disease. ${ }^{71}$ To comply with 
the definition of a risk factor, a reduction in heart rate by therapy should be associated with improved clinical outcomes. Several trials with ivabradine (the specific heart rate-reducing agent without any other known cardiovascular effects) have been conducted to explore this issue. Although no benefit was observed of heart rate reduction on outcomes in patients with stable coronary artery disease without clinical heart failure and with a heart rate of 70 beats/min or greater, ${ }^{72}$ a higher heart rate may serve as a risk factor for cardiovascular mortality and all-cause mortality in patients with left-ventricular systolic dysfunction with coronary artery disease and/or heart failure $^{73,74}$ (Appendix 5, www.cmaj.ca/lookup/suppl/ doi:10.1503/cmaj.150535/-/DC1). These findings suggest that the association of heart rate reduction with outcomes may differ among populations of different risk, and higher heart rate is due to different pathophysiological mechanisms in different conditions. $^{5}$

A linear relation was found between resting heart rate and risk of all-cause mortality and cardiovascular mortality, respectively. Among the included studies, only 3 studies described the dose-response relation continuously, ${ }^{13,34,64}$ and all indicated a linear relation. However, a J-shaped curve was also indicated by categorizing resting heart rate. ${ }^{44,48,55}$ Because this is a meta-analysis focusing on the general population (i.e., excluding studies in disease-specific populations), patients with a very low resting heart rate are likely to be more physically fit. Therefore, more benefit could be anticipated for patients with a very low resting heart rate. In addition, findings from trials involving patients with cardiovascular disease also indicated the possibility of "the slower the heart rate, the better." 75

\section{Limitations}

Our study had several limitations. First, measurement of resting heart rate is susceptible to numerous factors, ${ }^{76}$ and nighttime heart rate was found to be a better prognostic variable relative to resting heart rate and 24-hour heart rate; nighttime heart rate should be a better measure of heart rate because both the interference of sensory input as well as physical and mental activities were minimized. ${ }^{57}$ Second, patients with cardiovascular disease or subclinical cardiovascular disease with no antihypertensive treatment at baseline could have started taking heart rate-lowering medications during follow-up, and this would lead to an underestimation of the true magnitude of the observed associations. Third, although we extracted risks that reflected the greatest degree of control for potential confounders, the covariates adjusted and the extent to which they were adjusted varied in the original studies. Fourth, single factor did not successfully explain the substantial betweenstudy heterogeneity; however, meta-regression suggested that population characteristics and study designs might contribute in concrete to the heterogeneity. Finally, we searched for only English- and Chinese-language studies, and we found publication bias. However, the observed associations remained after incorporating theoretical missing studies.

\section{Conclusion}

Resting heart rate was associated with risk of allcause and cardiovascular mortality in the general population, independent of traditional cardiovascular risk factors. However, considering that the covariates adjusted for and the extent to which they were adjusted for varied in the included studies, as well as the presence of between-study heterogeneity and publication bias, collaborative pooling of individual participant data from prospective cohort studies is needed to better describe the association. Identification of the contribution of heart rate reduction on effects of cardiovascular events and mortality by other interventions like exercise with a clinical trial in the general population will provide evidence on whether heart rate is also a risk factor. Further studies are warranted to develop a prediction algorithm that would consider both resting heart rate and classic cardiovascular risk factors to allow physicians to use resting heart rate in clinical settings.

\section{References}

1. Dobre D, Borer JS, Fox K, et al. Heart rate: a prognostic factor and therapeutic target in chronic heart failure. The distinct roles of drugs with heart rate-lowering properties. Eur J Heart Fail 2014;16:76-85

2. Ohira T, Diez Roux AV, Prineas RJ, et al. Associations of psychosocial factors with heart rate and its short-term variability: multi-ethnic study of atherosclerosis. Psychosom Med 2008; 70:141-6.

3. Dinas PC, Koutedakis Y, Flouris AD. Effects of active and passive tobacco cigarette smoking on heart rate variability. Int J Cardiol 2013;163:109-15.

4. Mozaffarian D, Prineas RJ, Stein PK, et al. Dietary fish and n-3 fatty acid intake and cardiac electrocardiographic parameters in humans. J Am Coll Cardiol 2006;48:478-84.

5. Fox K, Borer JS, Camm AJ, et al. Resting heart rate in cardiovascular disease. J Am Coll Cardiol 2007;50:823-30.

6. Sajadieh A, Nielsen OW, Rasmussen V, et al. Increased heart rate and reduced heart-rate variability are associated with subclinical inflammation in middle-aged and elderly subjects with no apparent heart disease. Eur Heart J 2004;25:363-70.

7. Jensen MT, Marott JL, Allin KH, et al. Resting heart rate is associated with cardiovascular and all-cause mortality after adjusting for inflammatory markers: the Copenhagen City Heart Study. Eur J Prev Cardiol 2012;19:102-8.

8. Goff DC Jr, Lloyd-Jones DM, Bennett G, et al. 2013 ACC/ AHA guideline on the assessment of cardiovascular risk: a report of the American College of Cardiology/American Heart Association Task Force on Practice Guidelines. J Am Coll Cardiol 2014;63:2935-59.

9. European Guidelines on cardiovascular disease prevention in clinical practice (version 2012): the Fifth Joint Task Force of the European Society of Cardiology and Other Societies on Cardiovascular Disease Prevention in Clinical Practice (constituted by representatives of nine societies and by invited 
experts). Eur J Prev Cardiol 2012;19:585-667.

10. Leistner DM, Klotsche J, Palm S, et al. Resting heart rate as a tool for risk stratification in primary care: Does it provide incremental prognostic information? Eur J Prev Cardiol 2012; 19:275-84.

11. Morcet JF, Safar M, Thomas F, et al. Associations between heart rate and other risk factors in a large French population. $J$ Hypertens 1999;17:1671-6.

12. Cooney MT, Vartiainen E, Laatikainen T, et al. Elevated resting heart rate is an independent risk factor for cardiovascular disease in healthy men and women. Am Heart J 2010;159:612 619.e3.

13. Tverdal A, Hjellvik V, Selmer R. Heart rate and mortality from cardiovascular causes: a 12 year follow-up study of 379,843 men and women aged 40-45 years. Eur Heart J 2008;29:2772-81.

14. Stroup DF, Berlin JA, Morton SC, et al. Meta-analysis of observational studies in epidemiology: a proposal for reporting. Meta-analysis of observational studies in epidemiology (MOOSE) group. JAMA 2000;283:2008-12.

15. DerSimonian R, Laird N. Meta-analysis in clinical trials. Control Clin Trials 1986;7:177-88.

16. Higgins JP, Thompson SG, Deeks JJ, et al. Measuring inconsistency in meta-analyses. BMJ 2003;327:557-60.

17. Higgins JP, Thompson SG. Controlling the risk of spurious findings from meta-regression. Stat Med 2004;23:1663-82.

18. Egger M, Davey Smith G, Schneider M, et al. Bias in metaanalysis detected by a simple, graphical test. BMJ 1997; 315:629-34.

19. Duval S, Tweedie R. Trim and fill: a simple funnel-plot-based method of testing and adjusting for publication bias in metaanalysis. Biometrics 2000;56:455-63.

20. Wells GA, Shea B, O'Connell D, et al. The Newcastle-Ottawa Scale (NOS) for assessing the quality of nonrandomised studies in meta-analyses. Ottawa Hospital Research Institute. Available: www.ohri.ca/programs/clinical_epidemiology/oxford.asp (accessed 2015 Aug. 17).

21. Orsini N, Li R, Wolk A, et al. Meta-analysis for linear and nonlinear dose-response relations: examples, an evaluation of approximations, and software. Am J Epidemiol 2012;175:66-73.

22. Harrell FE Jr, Lee KL, Pollock BG. Regression models in clinical studies: determining relationships between predictors and response. J Natl Cancer Inst 1988;80:1198-202.

23. Orsini N, Bellocco R, Greenland S. Generalized least squares for trend estimation of summarized dose-response data. Stata J 2006;6:40-57.

24. Jackson D, White IR, Thompson SG. Extending DerSimonian and Laird's methodology to perform multivariate random effects meta-analyses. Stat Med 2010;29:1282-97.

25. Gillum RF, Makuc DM, Feldman JJ. Pulse rate, coronary heart disease, and death: the NHANES I Epidemiologic Follow-up Study. Am Heart J 1991;121:172-7.

26. Sandvik L, Erikssen J, Thaulow E, et al. Physical fitness as a predictor of mortality among healthy, middle-aged Norwegian men. N Engl J Med 1993;328:533-7.

27. Mensink GB, Hoffmeister H. The relationship between resting heart rate and all-cause, cardiovascular and cancer mortality. Eur Heart J 1997; 18:1404-10

28. Benetos A, Rudnichi A, Thomas F, et al. Influence of heart rate on mortality in a French population: role of age, gender, and blood pressure. Hypertension 1999;33:44-52.

29. Greenland P, Daviglus ML, Dyer AR, et al. Resting heart rate is a risk factor for cardiovascular and noncardiovascular mortality: the Chicago Heart Association Detection Project in Industry. Am J Epidemiol 1999;149:853-62.

30. Palatini P, Casiglia E, Julius S, et al. High heart rate: a risk factor for cardiovascular death in elderly men. Arch Intern Med 1999; 159:585-92.

31. Kristal-Boneh E, Silber H, Harari G, et al. The association of resting heart rate with cardiovascular, cancer and all-cause mortality. Eight year follow-up of 3527 male Israeli employees (the CORDrosoph Inf Serv Study). Eur Heart J 2000;21:116-24.

32. Reunanen A, Karjalainen J, Ristola P, et al. Heart rate and mortality. J Intern Med 2000;247:231-9.

33. Nilsson PM, Nilsson JA, Hedblad B, et al. Sleep disturbance in association with elevated pulse rate for prediction of mortality-consequences of mental strain? J Intern Med 2001; 250:521-9.

34. Seccareccia F, Pannozzo F, Dima F, et al. Heart rate as a predictor of mortality: the MATISS project. Am J Public Health 2001;91:1258-63.

35. Cheng YJ, Macera CA, Church TS, et al. Heart rate reserve as a predictor of cardiovascular and all-cause mortality in men. Med Sci Sports Exerc 2002;34:1873-8.

36. Kado DM, Lui LY, Cummings SR. Rapid resting heart rate: a simple and powerful predictor of osteoporotic fractures and mortality in older women. $J$ Am Geriatr Soc 2002;50:455-60.

37. Chang M, Havlik RJ, Corti MC, et al. Relation of heart rate at rest and mortality in the Women's Health and Aging Study. Am J Cardiol 2003;92:1294-9.

38. Hozawa A, Ohkubo T, Kikuya M, et al. Prognostic value of home heart rate for cardiovascular mortality in the general population: the Ohasama study. Am J Hypertens 2004;17:1005-10.

39. Okamura T, Hayakawa T, Kadowaki T, et al. Resting heart rate and cause-specific death in a 16.5-year cohort study of the Japanese general population. Am Heart J 2004;147:1024-32.

40. Fisher AA, Davis MW, Srikusalanukul W, et al. Does heart rate predict mortality in older, low-level care residents? Am J Geriatr Cardiol 2006;15:208-16.

41. Savonen KP, Lakka TA, Laukkanen JA, et al. Heart rate response during exercise test and cardiovascular mortality in middle-aged men. Eur Heart $J$ 2006;27:582-8.

42. Theobald H, Wandell PE. Effect of heart rate on long-term mortality among men and women. Acta Cardiol 2007;62:275-9.

43. Adabag AS, Grandits GA, Prineas RJ, et al. Relation of heart rate parameters during exercise test to sudden death and allcause mortality in asymptomatic men. Am J Cardiol 2008; 101:1437-43.

44. Hansen TW, Thijs L, Boggia J, et al. Prognostic value of ambulatory heart rate revisited in 6928 subjects from 6 populations. Hypertension 2008;52:229-35.

45. Kizilbash MA, Daviglus ML, Dyer AR, et al. Relation of heart rate with cardiovascular disease in normal-weight individuals: the Chicago Heart Association Detection Project in Industry. Prev Cardiol 2008;11:141-7.

46. Mai JZ, Zhao LC, Liu XQ, et al. [Association between heart rate and all-cause death and coronary event in the Chinese cohort: 16 years follow up results] [Article in Chinese]. Zhonghua Xin Xue Guan Bing Za Zhi 2009;37:750-3.

47. Batty GD, Shipley MJ, Kivimaki M, et al. Walking pace, leisure time physical activity, and resting heart rate in relation to disease-specific mortality in London: 40 years follow-up of the original Whitehall study. An update of our work with professor Jerry N. Morris (1910-2009). Ann Epidemiol 2010;20:661-9.

48. Nauman J, Nilsen TI, Wisloff U, et al. Combined effect of resting heart rate and physical activity on ischaemic heart disease: mortality follow-up in a population study (the HUNT study, Norway). J Epidemiol Community Health 2010;64:175-81.

49. Jensen MT, Marott JL, Jensen GB. Elevated resting heart rate is associated with greater risk of cardiovascular and all-cause mortality in current and former smokers. Int J Cardiol 2011; 151:148-54.

50. Jouven X, Escolano S, Celermajer D, et al. Heart rate and risk of cancer death in healthy men. PLOS ONE 2011;6:e21310.

51. Legeai $C$, Jouven $X$, Tafflet $M$, et al. Resting heart rate, mortality and future coronary heart disease in the elderly: the $3 \mathrm{C}$ Study. Eur J Cardiovasc Prev Rehabil 2011;18:488-97.

52. Nabi H, Kivimaki M, Empana JP, et al. Combined effects of depressive symptoms and resting heart rate on mortality: the Whitehall II prospective cohort study. J Clin Psychiatry 2011; 72:1199-206.

53. Nauman J, Janszky I, Vatten LJ, et al. Temporal changes in resting heart rate and deaths from ischemic heart disease. JAMA 2011;306:2579-87.

54. Inoue R, Ohkubo T, Kikuya M, et al. Predictive value for mortality of the double product at rest obtained by home blood pressure measurement: the Ohasama study. Am J Hypertens 2012;25:568-75.

55. Woodward M, Webster R, Murakami Y, et al. The association between resting heart rate, cardiovascular disease and mortality: evidence from 112,680 men and women in 12 cohorts. Eur J Prev Cardiol 2014;21:719-26.

56. Jensen MT, Suadicani P, Hein HO, et al. Elevated resting heart rate, physical fitness and all-cause mortality: a 16-year followup in the Copenhagen Male Study. Heart 2013;99:882-7.

57. Johansen CD, Olsen RH, Pedersen LR, et al. Resting, nighttime, and $24 \mathrm{~h}$ heart rate as markers of cardiovascular risk in middle-aged and elderly men and women with no apparent heart disease. Eur Heart J 2013;34:1732-9.

58. Pittaras AM, Faselis C, Doumas M, et al. Heart rate at rest, exercise capacity, and mortality risk in veterans. Am J Cardiol 2013;112:1605-9.

59. Plichart M, Thomas F, Empana JP, et al. Gender-specific trends in heart rate in the general population from 1992-2007: a study of 226,288 French adults. Eur J Prev Cardiol 2013; 20:61-72.

60. Saxena A, Minton D, Lee DC, et al. Protective role of resting heart rate on all-cause and cardiovascular disease mortality. Mayo Clin Proc 2013;88:1420-6.

61. Aladin AI, Whelton SP, Al-Mallah MH, et al. Relation of resting heart rate to risk for all-cause mortality by gender after 
considering exercise capacity (the Henry Ford exercise testing project). Am J Cardiol 2014;114:1701-6.

62. Floyd JS, Sitlani CM, Wiggins KL, et al. Variation in resting heart rate over 4 years and the risks of myocardial infarction and death among older adults. Heart 2015;101:132-8.

63. Hisamatsu T, Miura K, Ohkubo T, et al. High long-chain n-3 fatty acid intake attenuates the effect of high resting heart rate on cardiovascular mortality risk: a 24-year follow-up of Japanese general population. J Cardiol 2014;64:218-24.

64. Ho JE, Larson MG, Ghorbani A, et al. Long-term cardiovascular risks associated with an elevated heart rate: the Framingham Heart Study. J Am Heart Assoc 2014;3:e00668.

65. Ó Hartaigh B, Gill TM, Shah I, et al. Association between resting heart rate across the life course and all-cause mortality: longitudinal findings from the Medical Research Council (MRC) National Survey of Health and Development (NSHD). J Epidemiol Community Health 2014;68:883-9.

66. Ryu M, Gombojav B, Nam CM, et al. Modifying effects of resting heart rate on the association of binge drinking with allcause and cardiovascular mortality in older Korean men: the Kangwha Cohort Study. J Epidemiol 2014;24:274-80.

67. Wang A, Chen S, Wang C, et al. Resting heart rate and risk of cardiovascular diseases and all-cause death: the Kailuan study. PLOS ONE 2014;9:e110985.

68. Palatini P. Heart rate as a cardiovascular risk factor: Do women differ from men? Ann Med 2001;33:213-21.

69. Wannamethee G, Shaper AG, Macfarlane PW. Heart rate, physical activity, and mortality from cancer and other noncardiovascular diseases. Am J Epidemiol 1993;137:735-48.

70. Feiner JR, Finlay-Morreale HE, Toy P, et al. High oxygen partial pressure decreases anemia-induced heart rate increase equivalent to transfusion. Anesthesiology 2011;115:492-8.

71. Böhm M, Reil JC, Deedwania P, et al. Resting heart rate: risk indicator and emerging risk factor in cardiovascular disease. Am J Med 2015;128:219-28.

72. Fox K, Ford I, Steg PG, et al. Ivabradine in stable coronary artery disease without clinical heart failure. $N$ Engl J Med 2014; 371:1091-9.

73. Fox K, Komajda M, Ford I, et al. Effect of ivabradine in patients with left-ventricular systolic dysfunction: a pooled analysis of individual patient data from the BEAUTIFUL and SHIFT trials. Eur Heart J 2013;34:2263-70.

74. Böhm M, Swedberg K, Komajda M, et al. Heart rate as a risk factor in chronic heart failure (SHIFT): the association between heart rate and outcomes in a randomised placebo-controlled trial. Lancet 2010;376:886-94.

75. Reil JC, Bohm M. BEAUTIFUL results-The slower, the better? Lancet 2008;372:779-80.

76. Vogel CU, Wolpert C, Wehling M. How to measure heart rate? Eur J Clin Pharmacol 2004;60:461-6.

Affiliations: Departments of Epidemiology and Health Statistics (Zhang, Shen), and Clinical Medicine (Qi), Medical College of Qingdao University, Shandong, China.

Contributors: All of the authors made substantial contributions to study conception and design, acquisition of data, analysis and interpretation of data; and drafting of the article or revising it critically for important intellectual content. All of the authors gave final approval of the version to be published and agreed to act as guarantors of the work. 